\title{
Lower Bounds of Growth Order of Solutions of Schrödinger Equations with Homogeneous Potentials
}

\author{
By \\ Jun UCHiYAmA*
}

\section{§. Introduction}

In this paper we shall study the asymptotic behavior as $|x|$ tends to $\infty$ of a solution $u(x)$ of the following equation

$$
-\Delta u(x)+q(x) u(x)=\lambda u(x) \text { for } \quad x \in \Omega \subset R^{n},
$$

where $\lambda$ is a positive constant and $\Delta$ is the Laplacian. There are many articles investigating this problem for the two-particle Schrödinger operator. In these cases the authors usually assume that $q(x)$ tends to 0 as $|x|$ tends to $\infty$. However, here we turn our attention to the manyparticle Schrödinger operator, and so we assume that the potential $q(x)$ is a homogeneous function of $x$ of degree $-2 \gamma$. For example, the potential of the Schrödinger operator of an atom (or ion) consisting of a nucleus with charge $+Z$ and $m$ electrons given by

$$
q(x)=-\sum_{k=1}^{m} \frac{Z}{r_{k}}+\sum_{1 \leq k<l \leq m} \frac{1}{r_{k l}},
$$

where $r_{l}^{2}=\sum_{l=0}^{2}\left|x_{3 k-l}\right|^{2}, r_{k j}^{2}=\sum_{l=0}^{2}\left|x_{3 k-l}-x_{3 j-l}\right|^{2}$, is a homogeneous function of $x \in \mathbb{R}^{3 m}$ of degree -1 . This example shows us obviously that such a potential is not expected to decay uniformly at infinity, and also that the singular points of $q(x)$ spread out to infinity.

On this problem Weidmann [8] showed the fact that if $\Omega=\mathbb{R}^{n}$,

Communicated by S. Matsuura, February 8, 1974.

* Department of Mathematics, Kyoto University of Industrial Arsts and Textile Fibres, Kyoto. 
$0<\gamma \leqq \frac{1}{2}$ and $u(x) \in H^{2}\left(\mathbb{R}^{n}\right)$, then $u(x)$ must identically vanish in $\mathbb{R}^{n}$. Later on Weidmann [9] also showed the fact that if $\Omega=\mathbb{R}^{n}, 0<\gamma<1$ and $u(x) \in H^{2}\left(\mathbb{R}^{n}\right)$, then $u(x) \equiv 0$ in $\mathbb{R}^{n}$. These results mean that the Schrödinger operator $H=-\Delta+q(x)$ in $L_{2}\left(\mathbb{R}^{n}\right)$ has no positive eigenvalues. However, his treatment has two characteristics; the first one is that the problem is considered in the whole space $\mathbb{R}^{n}$, and the second one is that although he showed $u(x) \notin H^{2}\left(\mathbb{R}^{n}\right)$ for a not identically vanishing solution $u(x)$ of (1.1), he did not deduce it as a result of the growth estimates or lower bounds, as $|x|$ tends to $\infty$, of a not identically vanishing solution $u(x)$ itself of (1.1). However, we remark that if we study in detail the first paper [8] of Weidmann (by applying the method used in our paper, for example), his method leads us to the result that for any not identically vanishing solution $u(x) \in H^{2}{ }_{l o c}\left(\mathbb{R}^{n}\right)$ of $(1.1)$

$$
\liminf _{R \rightarrow \infty} R^{2 \gamma-1} \int_{|x| \leqq R}|u(x)|^{2} d x>0 \text {, in case } 0<\gamma<\frac{1}{2}
$$

and

$$
\liminf _{R \rightarrow \infty}(\log R)^{-1} \int_{|x| \leqq R}|u(x)|^{2} d x>0 \text {, in case } \gamma=\frac{1}{2}
$$

On the other hand Agmon ([1] and [2]) considered the equation (1.1) in $E_{R_{0}}=\left\{x \in \mathbb{R}^{n}|| x \mid>R_{0}\right\}$. He showed without detailed proof that if $\Omega=E_{R_{0}}$ and $\frac{1}{2} \leqq \gamma<1$, then any selfadjoint realization of $-\Delta+q(x)$ in $L_{2}\left(E_{R_{0}}\right)$ has no positive eigenvalues. His treatment is more general than Weidmann's ([8] and [9]) in the point that the domain $\Omega$ in which the equation (1.1) is satisfied is $E_{R_{0}}$, but more stringent restriction is given on $\gamma$. Here, however, we remark that Agmon [2] also deals with the case that $q(x)$ has no homogeneity. It seems to us that both Weidmann [8], [9] and Agmon [1], [2] showed only that $H=-\Delta+q(x)$ has no positive eigenvalues, but they did not give explicitly the lower bounds, as $|x|$ tends to $\infty$, for not identically vanishing solutions $u(x)$ of $(1.1)$.

Now we restrict ourselves to investigating the asymptotic behavior, as $|x|$ tends to $\infty$, of the solution $u(x)$ of (1.1) considered in $E_{R_{0}}$ or $R^{n}$. As a result, we shall have the fact that $H=-\Delta+q(x)$ has no positive 
eigenvalues. If $\Omega=E_{R_{0}}$ in (1.1), we can weaken the above mentioned condition on $\gamma$ given by Agmon [1] and [2] to the condition $\frac{1}{3}<\gamma \leqq 1$ (see Theorem 2.1). And if $\Omega=R^{n}$, we can have estimates stronger than (1.3) and (1.4). We have

$$
\liminf _{R \rightarrow \infty} R^{\gamma-1} \int_{R_{0} \leqq|x| \leqq R}|u(x)|^{2} d x>0,
$$

in case $\Omega=\mathbb{R}^{n}$ and $0<\gamma<1$ or in case $\Omega=E_{R_{0}}$ and $\frac{1}{3}<\gamma<1$, and

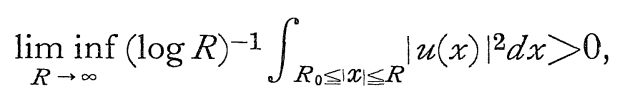

in case $\Omega=\mathbb{R}^{n}$ or $E_{R_{0}}$ and $\gamma=1$. Our method depends mainly on IkebeUchiyama [5], which may be said to be a compromise between Kato [6] and Roze [7], and partly on Agmon [3]. One point which should be noted in our treatment is way that in the course of our calculations we get rid of the influence of the singularities of the potential $q(x)$ spread out to infinity, in a way similar to the ones given by Weidmann [8] and Agmon [1], [2].

\section{§2. Notations and Summary}

Here we shall list the notation which will be used freely in the sequel (most of them have been the same ones used in Ikebe-Uchiyama [5]): $x=\left(x_{1}, \ldots, x_{n}\right)$ is a position vector in $\mathbb{R}^{n}$, where $n \geqq 3$;

$$
\begin{array}{ll}
|x|=\left(\left|x_{1}\right|^{2}+\ldots+\left|x_{n}\right|^{2}\right)^{1 / 2} ; & \\
\tilde{x}=\frac{x}{|x|}(|x| \neq 0) ; & \text { for } r>0 ; \\
S_{r}=\left\{x \in \mathbb{R}^{n}|| x \mid=r\right\} & \text { for } 0<s<r ; \\
B_{s r}=\left\{x \in \mathbb{R}^{n}|s<| x \mid<r\right\} & \text { for } r>0 ; \\
B_{r}=\left\{x \in \mathbb{R}^{n}|| x \mid \leqq r\right\} & \text { for } r>0 ; \\
E_{r}=\left\{x \in \mathbb{R}^{n}|| x \mid>r\right\} & \\
\partial_{i}=\frac{\partial}{\partial x_{i}} \quad(i=1, \ldots, n) ; &
\end{array}
$$


$D u=\left(\partial_{1} u(x), \ldots, \partial_{n} u(x)\right)$

$\langle f, g\rangle=\sum_{i=1}^{n} f_{i} g_{i} \quad$ for $\quad f=\left(f_{1}, \ldots, f_{n}\right) \in \boldsymbol{R}^{n}$ and

$g=\left(g_{1}, \ldots, g_{n}\right) \in \boldsymbol{R}^{n}$

$L_{2}$ denotes the class of square integrable functions, and thus $L_{2}\left(E_{R_{0}}\right)$ is all $L_{2}$ functions over $E_{R_{0}}$;

$H^{2}$ denotes the class of $L_{2}$ functions with distribution derivatives in $L_{2}$ up to the second order inclusive;

$L_{2}, l o c$ and $H_{l o c}^{2}$ denote the class of locally $L_{2}$ and $H^{2}$ functions, respectively;

$C^{m}$ denotes the class of $m$-times continuously differentiable functions; $Q_{\mu}\left(\boldsymbol{R}^{n}\right)$ denotes the class of functions $f(x)$ satisfying the "Stummel condition": if $n+\mu>4, \sup _{x \in R^{n}} \int_{|x-y| \leqq 1} \frac{|f(y)|^{2}}{|x-y|^{n-4+\mu}} d y<+\infty$, or if $n+\mu \leqq 4, \sup _{x \in \boldsymbol{R}^{n}} \int_{|x-y| \leqq 1}|f(y)|^{2} d y<+\infty$.

Now we shall state the conditions to be imposed on the potential $q(x)$ of the differential operator appearing on the left side of (1.1).

Assumption 1. The potential $q(x)$ is real valued and satisfies the following inequality: $|x|\langle D q, \tilde{x}\rangle \leqq(-2 \gamma) q(x)$ for $x \in \Omega$, where $\gamma$ is some constant. Here we remark that $\langle D q, \tilde{x}\rangle$ coincides with $\frac{\partial}{\partial|x|} q(x)$.

Assumption 2. For $q(x)$, we have $q(x) \in Q_{\mu}\left(\boldsymbol{R}^{n}\right)$ for some constant $\mu>0$.

Assumption 3. The unique continuation property holds.

Remark 2.1. If $q(x)$ is a homogeneous function of $x$ of degree $-2 \gamma$, then (2.1) is satisfied.

By a solution $u$ of equation (1.1) is meant an $H_{l o c}^{2}$, hence $L_{2}, l o c$, function which satisfies (1.1) in the distribution sense in the domain $\Omega$ in $\boldsymbol{R}^{n}$. 
Our aim is to prove the following theorems which restrict the asymptotic behavior of a non-trivial solution of (1.1).

Theorem 2.1. If $\Omega$ in (1.1) contains $E_{R_{0}}$ as an interior, and if $u$ is a not identically vanishing solution of (1.1) in $\Omega$ with a positive eigenvalue, $\lambda>0$, then we have

$$
\liminf _{R \rightarrow \infty} R^{\gamma-1} \int_{R_{0} \leqq|x| \leqq R}|u(x)|^{2} d x>0, \text { when } \frac{1}{3}<\gamma<1,
$$

and

$$
\liminf _{R \rightarrow \infty}(\log R)^{-1} \int_{R_{0} \leq|x| \leqq R}|u(x)|^{2} d x>0, \text { when } \gamma=1
$$

Corollary. If $\Omega$ satisfies the condition mentioned in the above theorem, and if $\frac{1}{3}<\gamma \leqq 1$, then a solution $u(x)$ of (1.1) in $\Omega$ with $\lambda>0$ which also belongs to $L_{2}(\Omega)$ must identically vanish in $\Omega$.

Remark 2.2. This corollary shows the fact that any selfadjoint realization in $L_{2}(\Omega)$ of the Schrödinger operator appearing on the left side of (1.1) has no positive eigenvalues.

Theorem 2.2. If $\Omega=\mathbb{R}^{n}$ in (1.1), and if $u$ is a not identically vanishing solution of (1.1) in $\mathbb{R}^{n}$ with a positive eigenvalue, $\lambda>0$, then we have

(2.4) $\quad \liminf _{R \rightarrow \infty} R^{\gamma-1} \int_{|x| \leq R}|u(x)|^{2} d x>0$, when $0<\gamma<1$,

and

$$
\liminf _{R \rightarrow \infty}(\log \mathrm{R})^{-1} \int_{|x| \leqq R}|u(x)|^{2} d x>0, \quad \text { when } \gamma=1 .
$$

Corollary. If $\Omega=\mathbb{R}^{n}$ in (1.1), and if $0<\gamma \leqq 1$, then a solution $u(x)$ of (1.1) in $\mathbb{R}^{n}$ with $\lambda>0$ which also belongs to $L_{2}\left(\mathbb{R}^{n}\right)$ must identically vanish in $\mathbb{R}^{n}$.

This corollary shows that Schrödinger operator investigated by us has the same property as the one mentioned in Remark 2.2.

We shall prove Theorem 2.1 in $\S 3$ and Theorem 2.2 in $\S 4$. In $\S 3$ 
we follow the procedures used in Ikebe-Uchiyama [5].

Finally we remark that even in the case where the potential $q(x)$ has the form $q(x)=q_{0}(x)+q_{1}(x)+q_{2}(x)$, where $q_{0}(x)$ satisfies the assumptions stated above, and both $q_{1}(x)$ and $q_{2}(x)$ are the potentials for the twoparticle system, namely $q_{1}(x)$ and $q_{2}(x)$ satisfy the conditions that $q_{1}(x)$ $=o\left(\frac{1}{|x|}\right), \frac{\partial}{\partial|x|} q_{2}(x)=o\left(\frac{1}{|x|}\right)$, and $q_{2}(x)=o(1)$ as $|x|$ tends to $\infty$, it would be possible to obtain a result which is similar to Theorem 2.1 mentioned above and which corresponds to Theorem 2.3 in Agmon [1]. We did not enter into this problem, however.

\section{§3. Proof of Theorem 2.1}

Let $u$ be a solution of (1.1):

$$
-\Delta u+q(x) u=\lambda u \quad \text { in } \Omega .
$$

By the reality of $q(x)$ assumed in Assumption 1, we can admit $u(x)$ to be a real valued function. If we introduce the function $v(x)$ by

$$
v(x)=|x|^{\frac{n-1}{2}} u(x)
$$

then the following result readily follows from (3.1).

Lemma 3.1. $v(x)$ satisfies the equation

$$
-\Delta v+\frac{n-1}{|x|}\langle D v, \tilde{x}\rangle+(q(x)+\tilde{q}(x)-\lambda) v(x)=0
$$

in $\Omega$, where

$$
\tilde{q}(x)=\frac{(n-1)(n-3)}{4|x|^{2}}
$$

What we want, in fact, to do for the present is to multiply (3.3) with $2|x|^{\alpha}\langle D v, \tilde{x}\rangle-k|x|^{\alpha-1} v$ and integrate over $B_{s r} \subset \Omega$. We will be led to Definition 3.2 by collecting the resulting surface integrals, and the mentioned integration (by parts) over $B_{s r}$ will be carried out in the proof of Lemma 3.3. 
Definition 3.2. Let $a$ and $k$ be real constants. We put for $r>0$ satisfying $S_{r} \subset \Omega$

$$
\begin{aligned}
F(r, \alpha ; k)= & \int_{S_{r}}\left\{2\left\langle D v^{\prime}, \tilde{x}\right\rangle^{2}-|D v|^{2}+(\lambda-q(x)-\tilde{q}(x))|v|^{2}\right. \\
& \left.-\frac{k}{|x|}\langle D v, \tilde{x}\rangle v+\frac{(\alpha+n-2)}{2} \frac{k}{|x|^{2}}|v|^{2}\right\}|x|^{\alpha} d S .
\end{aligned}
$$

Eemma 3.3. We have

$$
\begin{array}{r}
F(r, \alpha ; k)-F(s, \alpha ; k)=\int_{B_{s r}}\left[(3-a-n-k)\left(|D v|^{2}-\langle D v, \tilde{x}\rangle^{2}\right)\right. \\
+(\alpha+n-k-1)\langle D v, \tilde{x}\rangle^{2}+\{(a+n+k-1) \lambda-(a+n+k-1) q(x) \\
-|x|\langle D q, \tilde{x}\rangle-(a+n+k-3) \tilde{q}(x) \\
\left.\left.+\frac{k}{2}(\alpha+n-2)(\alpha+n-3) \frac{1}{|x|^{2}}\right\}|v|^{2}\right]|x|^{\alpha-1} d x
\end{array}
$$

for $r>s>0$, where $B_{s r} \subset \Omega$.

Proof. By Lemma 3.1 we have for $B_{s r} \subset \Omega$

$$
\begin{aligned}
\int_{B_{s r}}\left\{-\Delta v+\frac{n-1}{|x|}\right. & \langle D v, \tilde{x}\rangle+(q(x)+\tilde{q}(x)-\lambda) v(x)\} \\
& \times\left\{2|x|^{\alpha}\langle D v, \tilde{x}\rangle-k|x|^{\alpha-1} v(x)\right\} d x=0 .
\end{aligned}
$$

Applying the integration by parts, and then gathering the resulting surface integrals in the left-hand side and the volume integrals in the right-hand, we obtain the assertion.

Q.E.D.

Lemma 3.4. Let $\alpha_{0}=1+\gamma-n$. If $0 \leqq \gamma \leqq 1$, then we have for any $r \geqq s>0$ satisfying $B_{s r} \subset \Omega$

$$
F\left(r, \alpha_{0} ; \gamma\right) \geqq F\left(s, \alpha_{0} ; \gamma\right) .
$$

Proof. By Lemma 3.3 and Assumption 1 we have 


$$
\begin{aligned}
& F\left(r, \alpha_{0} ; \gamma\right)-F\left(s, \alpha_{0} ; \gamma\right) \\
& \geqq \int_{B_{s}}\left[2(1-\gamma)\left(|D v|^{2}-\langle D v, \tilde{x}\rangle^{2}\right)+\{2 \gamma \lambda+2(1-\gamma) \tilde{q}(x)\right. \\
& \left.\left.\quad+\frac{1}{2}(2-\gamma)(1-\gamma) \gamma \frac{1}{|x|^{2}}\right\}|v|^{2}\right]|x|^{\alpha_{0}-1} d x
\end{aligned}
$$

According to $|D v|^{2} \geqq\langle D v, \tilde{x}\rangle^{2}$ and $0 \leqq \gamma \leqq 1$, we have the statement.

Q.E.D.

In the sequel of this section, we assume that the conditions given in Theorem 2.1 hold, namely that $\Omega \supset \overline{E_{R_{0}}}$ and

$$
\frac{1}{3}<\gamma \leqq 1
$$

Our next task is to estimate from below the functional $F\left(r, a_{0} ; \gamma\right)$ itself when $v[u]$ is a non-trivial solution of $(3.3)[(3.1)]$. To this end we further introduce the function $w_{m}(x)$ for a real constant $m$ and a real-valued function $f(t) \in C^{3}\left(R_{0}, \infty\right)$ by

$$
w_{m}(x)=e^{m f(|x|)} v(x)
$$

The equation fulfiled by $w_{m}(x)$ can be easily derived from (3.3) and (3.10), that is, we have

Lemma 3.5. In $E_{R_{0}} w_{m}(x)$ satisfies the equation

$$
\begin{aligned}
& -\Delta w_{m}+\left(\frac{n-1}{|x|}+2 m f^{\prime}(|x|)\right)\left\langle D w_{m}, \tilde{x}\right\rangle+(q(x)+\tilde{q}(x)-\lambda \\
& \left.-m^{2}\left(f^{\prime}(|x|)\right)^{2}+m f^{\prime \prime}(|x|)\right) w_{m}(x)=0 .
\end{aligned}
$$

We shall multiply (3.10) with $2|x|^{\alpha}\left\langle D w_{m}, \tilde{x}\right\rangle-k|x|^{\alpha-1} v(x)$ and integrate by parts over $B_{s r}$, which will be an essential part of the proof of Lemma 3.8. The resulting surface integrals plus an additional surface integral form the following functional.

Definition 3.6. Let $m, \alpha, \beta$ and $k$ be real constants, and let $f(t)$, $t>R_{0}$, be a real-valued $C^{3}$ function. We put for $r>R_{0}$ 


$$
\begin{aligned}
& F(r, \alpha, \beta, m ; k ; f(r)) \\
& =\int_{S_{r}}\left[2\left\langle D w_{m}, \tilde{x}\right\rangle^{2}-\left|D w_{m}\right|^{2}-\frac{k}{|x|}\left\langle D w_{m}, \tilde{x}\right\rangle_{m}\right. \\
& \quad+\left\{\lambda-q(x)-\tilde{q}(x)+m^{2} f^{\prime 2}-m f^{\prime \prime}-|x|^{\beta-\alpha}\right. \\
& \left.\left.\quad+\frac{k}{2}(\alpha+n-2) \frac{1}{|x|^{2}}+\frac{k m}{|x|} f^{\prime}\right\}\left|w_{m}\right|^{2}\right]|x|^{\alpha} d S .
\end{aligned}
$$

Here we remark a relation between $F(r, \alpha, \beta, m ; k ; f)$ and $F(r, \alpha ; k)$.

Lemma 3.7. We have for $r>R_{0}$

$$
\begin{aligned}
& F(r, \alpha, \beta, m ; k ; f)=e^{2 m f(r)}\left[F(r, \alpha ; k)+\int_{S_{r}}\left\{2 m f^{\prime}\langle D v, \tilde{x}\rangle v\right.\right. \\
& \left.\left.\quad+\left(2 m^{2} f^{\prime 2}-m f^{\prime \prime}-|x|^{\beta-\alpha}\right)|v|^{2}\right\}\right]|x|^{\alpha} d S .
\end{aligned}
$$

Proof. By (3.5), (3.10) and (3.12), we have easily the assertion. Q.E.D.

Lemma 3.8. We have for $r>s>R_{0}$

$$
\begin{aligned}
& F(r, \alpha, \beta, m ; k ; f)-F(s, \alpha, \beta, m ; k ; f) \\
& \quad=\int_{B_{s} r}\left[(3-\alpha-n-k)\left(\left|D w_{m}\right|^{2}-\left\langle D w_{m}, \tilde{x}\right\rangle^{2}\right)\right. \\
& \quad+\left(\alpha+n-k-1+4 m|x| f^{\prime}\right)\left\langle D w_{m}, \tilde{x}\right\rangle^{2}-2|x|^{\beta-\alpha+1}\left\langle D w_{m}, \tilde{x}\right\rangle w_{m} \\
& \quad+\{(\alpha+n+k-1) \lambda-(\alpha+n+k-1) q(x)-|x|\langle D q, \tilde{x}\rangle \\
& \quad-(\alpha+n+k-3) \tilde{q}(x)+(\alpha+n+k-1) m^{2} f^{\prime 2}+2 m^{2}|x| f^{\prime} f^{\prime \prime} \\
& \quad-(\alpha+n-1) m f^{\prime \prime}+k(\alpha+n-2) \frac{1}{|x|} m f^{\prime}-|x| m f^{\prime \prime \prime} \\
& \quad-(\beta+n-1)|x|^{\beta-\alpha} \\
& \left.\left.\quad+\frac{k}{2}(\alpha+n-2)(\alpha+n-3) \frac{1}{|x|^{2}}\right\}\left|w_{m}\right|^{2}\right]|x|^{\alpha-1} d x .
\end{aligned}
$$

Proof. We first note the following identity which follows by integration by parts:

$$
2 \int_{B_{s r}}\langle D w, \tilde{x}\rangle w|x|^{\beta} d x=\int_{S_{r}}|w|^{2}|x|^{\beta} d S-\int_{S_{s}}|w|^{2}|x|^{\beta} d S
$$




$$
-(n+\beta-1) \int_{B_{s r}}|w|^{2}|x|^{\beta-1} d x .
$$

By Lemma 3.5 and (3.15) with $w=w_{m}$ we have for $R_{0}<s<r$

$$
\begin{aligned}
& \int_{B_{s r}}\left\{-\Delta w_{m}+\left(\frac{n-1}{|x|}+2 m f^{\prime}\right)\left\langle D w_{m}, \tilde{x}\right\rangle+(q(x)+\tilde{q}(x)-\lambda\right. \\
& \left.\left.-m^{2} f^{\prime 2}+m f^{\prime \prime}\right) w_{m}\right\}\left\{2|x|^{\alpha}\left\langle D w_{m}, \tilde{x}\right\rangle-k|x|^{\alpha-1} w_{m}\right\} d x \\
& \quad-2 \int_{B_{s r}}\left\langle D w_{m}, \tilde{x}\right\rangle w_{m}|x|^{\beta} d x-(n+\beta-1) \int_{B_{s r}}\left|w_{m}\right|^{2}|x|^{\beta-1} d x \\
& \quad+\int_{S_{r}}\left|w_{m}\right|^{2}|x|^{\beta} d S-\int_{S_{s}}\left|w_{m}\right|^{2}|x|^{\beta} d S=0 .
\end{aligned}
$$

Now we perform integration by parts as in the proof of Lemma 3.3, and we have the assertion.

Q.E.D.

Let $\eta$ be a constant to satisfy

$$
\gamma-\frac{1}{3}>\eta>0
$$

We put

$$
\begin{aligned}
& \varepsilon=1-\gamma+\eta, \\
& \delta=\frac{1}{2}(1+\gamma-\eta),
\end{aligned}
$$

and

$$
f(r)=r^{\varepsilon}
$$

Lemima 3.9. There exist some $R_{1}>R_{0}$ and some $m_{0}>0$ such that for any $r>s \geqq R_{1}$ and for any $m \geqq m_{0}$ we have

$$
F\left(r, \alpha_{0}, \alpha_{0}-\delta, m ; \gamma ; r^{\varepsilon}\right) \geqq F\left(s, \alpha_{0}, \alpha_{0}-\delta, m ; \gamma ; s^{\varepsilon}\right),
$$

where $\alpha_{0}$ is given in Lemma 3.4.

Proof. We put in Lemma $3.8 \alpha=\alpha_{0}=1-n+\gamma, \beta=\alpha_{0}-\delta$ and $f(r)=r^{\varepsilon}$. Then by the inequality

$$
\left.\left.|2| x\right|^{1-\delta}\left\langle D w_{m}, \tilde{x}\right\rangle w_{m}\left|\leqq \frac{1}{\gamma \lambda}\right| x\right|^{2(1-\delta)}\left\langle D w_{m}, \tilde{x}\right\rangle^{2}+\gamma \lambda\left|w_{m}\right|^{2}
$$


and by Assumption 1 we have

$$
\begin{aligned}
& F\left(r, \alpha_{0}, \alpha_{0}-\delta, m ; \gamma ; r^{\varepsilon}\right)-F\left(s, \alpha_{0}, \alpha_{0}-\delta, m ; \gamma ; s^{\varepsilon}\right) \\
& \quad \geqq \int_{B_{s} r}\left[2(1-\gamma)\left(\left|D w_{m}\right|^{2}-\left\langle D w_{m}, \tilde{x}\right\rangle^{2}\right)\right. \\
& \quad+\left(4 m \varepsilon|x|^{\varepsilon}-\frac{1}{\gamma \lambda}|x|^{2(1-\delta)}\right)\left\langle D w_{m}, \tilde{x}\right\rangle^{2} \\
& \quad+\left\{\gamma \lambda+2(1-\gamma) \tilde{q}(x)+|x|^{2(\varepsilon-1)}\left\{2 \varepsilon^{2} m^{2}(\varepsilon+\gamma-1)\right.\right. \\
& \left.\quad-\varepsilon m|x|^{-\varepsilon}\left(\varepsilon^{2}-\gamma^{2}+\gamma \varepsilon-3 \varepsilon+2\right)\right\}-(\gamma-\delta)|x|^{-\delta} \\
& \left.\left.\quad+\frac{1}{2} \gamma(2-\gamma)(1-\gamma) \frac{1}{|x|^{2}}\right\}\left|w_{m}\right|^{2}\right]|x|^{\alpha_{0}-1} d x .
\end{aligned}
$$

By (3.16), (3.17) and (3.18), we have

$$
\begin{aligned}
& \varepsilon>0, \\
& \varepsilon>1-\gamma, \\
& \delta>0,
\end{aligned}
$$

and

$$
\varepsilon+2 \delta=2
$$

Now let $m_{0}=\frac{1}{4 \varepsilon \gamma \lambda}$ and choose $R_{1}$ sufficiently large. Then we have the assertion easily.

Q.E.D.

Lemma 3.10. Assume that the solution $u$ (and hence $v$ ) is not identically equal to 0 in $\Omega$. Then there exists some sequence $\left\{r_{l}\right\}_{l=1,2, \ldots}$ such that $R_{1}<r_{1}<r_{2}<\ldots<r_{l}<r_{l+1}<\ldots, \lim _{l \rightarrow \infty} r_{l}=\infty$ and

$$
\int_{S_{r_{l}}}|v|^{2} d S>0 \quad(l=1,2, \ldots)
$$

Proof. If we assume that the above statement is false, then there exists some $r_{0} \geqq R_{1}$ such that $\int_{S_{r}}|v|^{2} d S=0$ for any $r \geqq r_{0}$. Then we have $v(x)$ and hence $u(x)$ must vanish identically in $E_{r_{0}}$ which by Assumption 3 leads to $u(x) \equiv 0$ in $\Omega$. This is a contradiction.

Q.E.D. 
Lemma 3.11. Let $R_{2}=r_{1}$, where $r_{1}$ is given in Lemma 3.10. Then there exists some constant $m_{1} \geqq m_{0}$ such that

$$
F\left(R_{2}, \alpha_{0}, \alpha_{0}-\delta, m_{1} ; \gamma ; R_{2}^{\varepsilon}\right)>0 \text {. }
$$

Proof. By Lemma 3.7 we have

$$
\begin{aligned}
& F\left(R_{2}, \alpha_{0}, \alpha_{0}-\delta, m ; \gamma ; R_{2}^{\varepsilon}\right)=\varepsilon^{2 m R_{2}^{\varepsilon}}\left[F\left(R_{2}, \alpha_{0} ; \gamma\right)\right. \\
& \quad+\int_{S_{R 2}}\left\{2 m^{2} \varepsilon^{2} R_{2}^{2(\varepsilon-1)}|v|^{2}+m \varepsilon\left(2 R_{2}^{\varepsilon-1}\langle D v, \tilde{x}\rangle v\right.\right. \\
& \left.\left.\left.\quad-(\varepsilon-1) R_{2}^{\varepsilon-2}|v|^{2}\right)-|x|^{-\delta}|v|^{2}\right\} R_{2}^{\alpha_{0}} d S\right] .
\end{aligned}
$$

Now in the right hand side of (3.27) the coefficient of $e^{2 m R_{2}^{\varepsilon}}$ is a quadratic form in $m$, in which the coefficient of $m^{2}$ is positive. Then we have the assertion.

Q.E.D.

Lemma 3.12. Let $R_{2}$ and $m_{1}$ be as in Lemma 3.11. Then we have

$$
F\left(r, \alpha_{0}, \alpha_{0}-\delta, m_{1} ; \gamma ; r^{\varepsilon}\right)>0 \quad \text { for } \quad r \geqq R_{2}
$$

Proof. Since $R_{2} \geqq R_{1}$, the assertion is an immediate consequence of Lemmas 3.9 and 3.11 .

Q.E.D.

Lemma 3.13. Either Case I or Case II and only one of them holds, where

Case I: There exists some sequence $\left\{r_{l^{\prime}}\right\}_{l=1,2}, \ldots$ such that $R_{2}<r_{1^{\prime}}<r_{2^{\prime}}<$ $<r_{l^{\prime}}<r_{l^{\prime}+1}<\ldots, \lim _{l \rightarrow \infty} r_{l^{\prime}}=\infty$ and

$$
\int_{S_{r^{\prime}}}\langle D v, \tilde{x}\rangle v d S \leqq 0 \quad(l=1,2, \ldots)
$$

and

Case II: $\quad$ There exists some $R_{3} \geqq R_{2}$ such that

$$
\int_{S_{r}}\langle D v, \tilde{x}\rangle v d S>0 \quad \text { for any } \quad r \geqq R_{3} .
$$

Proof. Obvious.

Q.E.D. 
Lemma 3.14. If Case I in Lemma 3.13 holds, then there exists some $R_{4} \geqq R_{2}$ such that

$$
F\left(R_{4}, \alpha_{0} ; \gamma\right)>0
$$

Proof. We have by Lemma 3.12 .

$$
F\left(r^{\prime}, a_{0}, \alpha_{0}-\delta, m_{1} ; \gamma ; r_{l^{\prime}}^{\varepsilon}\right)>0 \quad(l=1,2, \ldots)
$$

Moreover by Lemma 3.7, we have

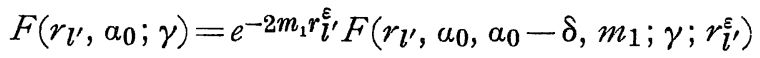

$$
\begin{aligned}
& -r_{l^{\prime}}^{\alpha_{0}+\varepsilon-1} \int_{S_{r_{l^{\prime}}}} 2 m_{1} \varepsilon\langle D v, \tilde{x}\rangle v d S+\left(r_{l^{\prime}}^{-\delta}+m_{1} \varepsilon(\varepsilon-1) r_{l^{\prime}}^{\varepsilon-2}\right. \\
& \left.-2 m_{1}^{2} \varepsilon^{2} r_{l^{\prime}}^{2 \varepsilon-2}\right) r_{l^{\prime}}^{\alpha_{0}} \int_{S_{r_{l^{\prime}}}}|v|^{2} d S
\end{aligned}
$$

Here we remark that

$$
2 \varepsilon-2<-\delta
$$

In fact by (3.16), (3.17) and (3.18) we have

$$
2 \varepsilon-2+\delta=\frac{3}{2}\left(\frac{1}{3}+\eta-\gamma\right)<0 .
$$

Now by $(3.30)$ we can choose $l_{0}$ large enough to satisfy

$$
r_{l_{0}^{\prime}}^{-\delta}+m_{1} \varepsilon(\varepsilon-1) r_{l_{0}^{\prime}}^{\varepsilon-2}-2 m_{1}^{2} \varepsilon^{2} r_{l_{0}^{\prime}}^{2 \varepsilon-2}>0
$$

Putting $R_{4}=r_{l_{0}^{\prime}}$, we have the statement of Lemma 3.14. Q.E.D.

Lemma 3.15. If Case I in Lemma 3.13 holds, then there exists the limit ( $\infty$ permitted) of $F\left(r, \alpha_{0} ; \gamma\right)$ as $r \rightarrow \infty$ and its value is positive.

Proof. By Lemmas 3.4 and 3.14 we have

$$
F\left(r, \alpha_{0} ; \gamma\right) \geqq F\left(R_{4}, \alpha_{0} ; \gamma\right)>0 \quad \text { for } \quad r \geqq R_{4}
$$

Moreover Lemma 3.4 tells that $F\left(r, \alpha_{0} ; \gamma\right)$ is a monotone increasing function of $r$. These imply that the assertion holds.

Q.E.D. 
Here we insert a property of the solution $u(x)$ of the equation (3.1) which holds under an assumption less restrictive than the one assumed in Theorem 2.1. Then we apply the following result to the proofs of Theorems 2.1 and 2.2 .

Lemma 3.16. If $q(x)$ in (3.1) satisfies Assumption 2, and if $\Omega \supset E_{R_{0}}$ then there exists some constant $C>0$ such that for any $r \geqq s>R_{0}+1$ we have

$$
\int_{B_{s r}}|D u|^{2} d x \leqq C \int_{B_{s-1, r+1}}|u|^{2} d x
$$

Proof. Let $\zeta_{s r}(t) \in C^{2}(0, \infty)$ satisfy the following conditions: $0 \leqq \zeta_{s r}(t)$ $\leqq 1$ for $t \in(0, \infty), \zeta_{s r}(t)=0$ for $t \in(0, s-1)$ and $t \in(r+1, \infty), \zeta_{s r}(t)=1$ for $t \in(s, r)$, and $\left|\zeta_{s r}^{\prime}(t)\right| \leqq C_{1},\left|\zeta_{s r}^{\prime \prime}(t)\right| \leqq C_{1}$ for $t \in(0, \infty)$ where $C_{1}$ is a constant independent of $s$ and $r$. Then by integration by parts and by (3.1) we have

$$
\begin{aligned}
& \int_{B_{s-1}, r+1}\left(\zeta_{s r}(|x|)\right)^{2}\left\{2|D u|^{2}+2(q(x)-\lambda)|u|^{2}\right\} d x \\
= & \int_{B_{s-1}, r+1} \zeta_{s r}^{2}\left(2|D u|^{2}+2 \Delta u \cdot u\right) d x=\int_{B_{s-1}, r+1} \zeta_{s r}^{2} \Delta\left(|u|^{2}\right) d x \\
= & \int_{B_{s-1}, r+1} \Delta\left(\zeta_{s r}^{2}\right)|u|^{2} d x \\
= & \int_{B_{s-1}, r+1}\left(2 \zeta_{s r}^{\prime 2}+2 \zeta_{s r} \zeta_{s r}^{\prime \prime}+2(n-1) \zeta_{s r} \zeta_{s r}^{\prime} \frac{1}{|x|}\right)|u|^{2} d x .
\end{aligned}
$$

On the other hand for the potential $q(x)$ satisfying Assumption 2, IkebeKato [4] proves the fact that for any $\varepsilon>0$ there exists some constant $C_{\varepsilon}=$ $C(\varepsilon)>0$ such that for any $\varphi(x) \in H^{2}\left(\mathbb{R}^{n}\right)$

$$
\int_{\mathbb{R}^{n}}|q(x)||\varphi(x)|^{2} d x \leqq \varepsilon \int_{\mathbb{R}^{n}}|D \varphi(x)|^{2} d x+C_{\varepsilon} \int_{\mathbb{R}^{n}}|\varphi(x)|^{2} d x
$$

In the above put $\varphi(x)=\zeta_{s r}(x) u(x) \in H^{2}\left(\mathbb{R}^{n}\right)$. Then we have

$$
\begin{aligned}
& \int_{B_{s-1}, r+1}|q(x)|\left|\zeta_{s r} u\right|^{2} d x \leqq \varepsilon \int_{B_{s-1}, r+1}\left|D\left(\zeta_{s r} u\right)\right|^{2} d x \\
& \quad+C_{\varepsilon} \int_{B_{s-1}, r+1}\left|\zeta_{s r} u\right|^{2} d x \leqq 2 \varepsilon \int_{B_{s-1}, r+1} \zeta_{s r}^{2}|D u|^{2} d x
\end{aligned}
$$




$$
+\int_{B_{s-1}, r+1}\left(2 \varepsilon \zeta_{s r}^{\prime 2}+C_{\varepsilon} \zeta_{s r}^{2}\right)|u|^{2} d x
$$

Therefore by (3.33) and (3.35), we have

$$
\begin{aligned}
& (1-2 \varepsilon) \int_{B_{s-1}, r+1} \zeta_{s r}^{2}|D u|^{2} d x \\
& \quad \leqq \int_{B_{s-1}, r+1}\left\{\lambda \zeta_{s r}^{2}+2 \varepsilon \zeta_{s r}^{\prime 2}+C_{\varepsilon} \zeta_{s r}^{2}+\zeta_{s r}^{\prime 2}+\zeta_{s r} \zeta_{s r}^{\prime \prime}\right. \\
& \left.\quad+(n-1) \zeta_{s r} \zeta_{s r}^{\prime} \frac{1}{|x|}\right\}|u|^{2} d x \\
& \quad \leqq\left\{\lambda+C_{\varepsilon}+(2 \varepsilon+1) C_{1}^{2}+C_{1}+(n-1) C_{1} R_{0}^{-1}\right\} \int_{B_{s-1}, r+1}|u|^{2} d x
\end{aligned}
$$

If $0<\varepsilon<\frac{1}{2}$, we have

$$
\int_{B_{s r}}|D u|^{2} d x \leqq \int_{B_{s-1, r+1}} \zeta_{s r}^{2}|D u|^{2} d x \leqq C \int_{B_{s-1}, r+1}|u|^{2} d x
$$

where $C=\frac{1}{1-2 \varepsilon}\left\{\lambda+C_{\varepsilon}+(2 \varepsilon+1) C_{1}^{2}+C_{1}+(n-1) C_{1} R_{0}^{-1}\right\}$, which shows that Lemma 3.16 holds.

Q.E.D.

Having prepared all the necessary tools for proving Theorem 2.1, we now proceed to the

Proof of Theorem 2.1. In the sequel, let us regard $C_{i}(i=2,3, \ldots)$ as positive constants independent of $s$ and $r$ satisfying $r>s>R_{0}$, where $\bar{E}_{R_{0}} \subset \Omega$. Firstly we assume that Case I in Lemma 3.13 holds. By $v(x)$ $=|x|^{\frac{n-1}{2}} u(x)$ and by Definition 3.2, we have

$$
\begin{aligned}
& F\left(r, \alpha_{0} ; \gamma\right)=r \gamma \int_{S_{r}}\left[2\langle D u, \tilde{x}\rangle^{2}-|D u|^{2}+\frac{(n-1-\gamma)}{|x|}\langle D u, \tilde{x}\rangle u\right. \\
& \left.\quad+(\lambda-q(x))|u|^{2}+\frac{(1-\gamma)(n-1-\gamma)}{2|x|^{2}}|u|^{2}\right] d S,
\end{aligned}
$$

where $\alpha_{0}=1+\gamma-n$ given in Lemma 3.4. By Lemma 3.15, there exist some $C_{2}>0$ and some $R_{5}>R_{0}$ such that for any $r>R_{5}$ we have

$$
\int_{S_{r}}\left[2\langle D u, \tilde{x}\rangle^{2}-|D u|^{2}+\frac{(n-1-\gamma)}{|x|}\langle D u, \tilde{x}\rangle u\right.
$$




$$
\left.+(\lambda-q(x))|u|^{2}+\frac{(1-\gamma)(n-1-\gamma)}{2|x|^{2}}|u|^{2}\right] d S \geqq C_{02} \gamma^{-r}
$$

This shows

$$
\int_{S_{r}}\left[\left.\left|D u^{2}+\right| u\right|^{2}+|q(x)||u|^{2}\right] d S \geqq C_{3} r^{-r} \quad \text { for any } r>R_{5}
$$

Multiply both sides of (3.39) by $\zeta_{s r}(|x|)^{2}$, which was defined in the proof of Lemma 3.16, and integrate with respect to $r$ from $s-1$ to $r+1$, where $R_{5}+2<s<r$. Then we have

$$
\begin{aligned}
& \int_{B_{s-1}, r+1}\left(\zeta_{s r}^{2}|D u|^{2}+\left|\zeta_{s r} u\right|^{2}+|q(x)|\left|\zeta_{s r} u\right|^{2}\right) d x \\
& \geqq\left\{\begin{array}{lll}
\frac{C_{3}}{1-\gamma}\left(r^{1-\gamma}-s^{1-\gamma}\right), & \text { when } & \frac{1}{3}<\gamma<1, \\
C_{3}(\log r-\log s), & \text { when } \quad \gamma=1 .
\end{array}\right.
\end{aligned}
$$

In a fashion similar to the proof of Lemma 3.16, using Lemma 3.16, we can estimate the left side of (3.40) as follows:

$$
\begin{aligned}
& \text { (left side of }(3.40)) \leqq C_{4} \int_{B_{s-1}, r+1}\left\{|D u|^{2}+|u|^{2}\right\} d x \\
& \leqq C_{4}\left\{C \int_{B_{s-2, r+2}}|u|^{2} d x+\int_{B_{s-1, r+1}}|u|^{2} d x\right\} \leqq C_{5} \int_{B_{s-2, r+2}}|u|^{2} d x
\end{aligned}
$$

Thus we have

$$
C_{5} \int_{B_{s-2, \gamma+2}}|u|^{2} d x \geqq\left\{\begin{array}{lll}
\frac{C_{3}}{1-\gamma}\left(\gamma^{1-\gamma}-s^{1-\gamma}\right), & \text { when } & \frac{1}{3}<\gamma<1, \\
C_{3}(\log \gamma-\log s), & \text { when } \quad \gamma=1 .
\end{array}\right.
$$

Therefore we have the assertion of Theorem 2.1.

Secondly we assume that Case II in Lemma 3.13 holds. We then have

$$
\left.\frac{d}{d r}\left(\int_{S_{r}}|u|^{2} d S\right)=\frac{d}{d r}\left(\int_{S_{r}}|v|^{2}|x|^{1-n} d S\right)=2 r^{1-n} \int_{S_{r}}\langle D v, \tilde{x}\rangle v d S\right\rangle 0
$$

for any $r>R_{3}$. This shows that $\int_{S_{r}}|u|^{2} d S$ is a monotone increasing function of $r$ in $\left(R_{3}, \infty\right)$. Since $u(x)$ is not identically null in $\Omega$ and the unique continuation property holds, there exist some $R_{\mathbf{6}} \geqq R_{\mathbf{3}}$ and some 
$C_{6}>0$ such that for any $r \geqq R_{6}$

$$
\int_{S_{r}}|u|^{2} d S \geqq C_{6},
$$

where $C_{6}=\int_{S_{R 6}}|u|^{2} d S>0$. Thus we have

$$
\liminf _{R \rightarrow \infty} R^{-1} \int_{R_{0} \leqq|x| \leqq R}|u|^{2} d x \geqq C_{6}>0 .
$$

Also in this case, noting $\gamma>0$, we have the assertion of Theorem 2.1.

Q.E.D.

Proof of Corollary to Theorem 2.1. Obvious from Theorem 2.1.

Q.E.D.

\section{$\S 4$. Proof of Theorem 2.2}

Hereafter we shall consider the equation

$$
-\Delta u(x)+q(x) u(x)=\lambda u(x)
$$

in the whole space $\boldsymbol{R}^{n}$ where $n \geqq 3$. In this section we assume that conditions in Theorem 2.2 holds. The main purpose of this section is to prove

$$
F\left(r, \alpha_{0} ; \gamma\right)>0
$$

for some $r$ under the restriction $0<\gamma \leqq 1$ weaker than the one $\frac{1}{3}<\gamma \leqq 1$ which is assumed in $\S 3$. Now we shall start with proving the following Lemma.

Lemma 4.1. There exists some sequence $\left\{s_{l}\right\}_{l=1,2, \ldots}$ such that $\lim _{l \rightarrow \infty} s_{l}=0$ and

$$
\lim _{l \rightarrow \infty} F\left(s_{l}, \alpha_{0} ; \gamma\right)=0
$$

Proof. Let $p(x) \in Q_{\mu}\left(\boldsymbol{R}^{n}\right)$ for some $\mu>0$. By Ikebe-Kato [4] there exists some constant $C>0$ such that for any $\varphi(x) \in H^{2}\left(\mathbb{R}^{n}\right)$ and $\psi(x)$ $\in H^{1}\left(\boldsymbol{R}^{n}\right)$ we have 


$$
\int_{R^{n}}|p(x) \varphi(x)|^{2} d x \leqq C \int_{R^{n}}\left\{|\Delta \varphi(x)|^{2}+|\varphi(x)|^{2}\right\} d x
$$

and

$$
\int_{\boldsymbol{R}^{n}}|p(x)||\psi(x)|^{2} d x \leqq C \int_{\boldsymbol{R}^{n}}\left\{|D \psi(x)|^{2}+|\psi(x)|^{2}\right\} d x .
$$

Then we have for $u(x) \in H_{l o c}^{2}\left(\boldsymbol{R}^{n}\right)$

$$
\int_{|x| \leq 1}|p(x) u(x)|^{2} d x<+\infty
$$

and

$$
\int_{|x| \leq 1}|p(x)|\left|\partial_{i} u(x)\right|^{2} d x<+\infty
$$

for $i=1,2, \ldots, n$. These show

$$
\liminf _{s \rightarrow 0} s \int_{S_{s}}|p(x) u(x)|^{2} d S=0
$$

and

$$
\liminf _{s \rightarrow 0} s \int_{S_{s}}|p(x)|\left|\partial_{i} u(x)\right|^{2} d S=0 .
$$

Noting that $\mid x^{\mid \varepsilon-\frac{3}{2}} \in Q_{\mu}\left(\boldsymbol{R}^{n}\right)$ and $\left(\mid q(x) \| x^{\mid \varepsilon-\frac{3}{2}}\right)^{\frac{1}{2}} \in Q_{\mu}\left(\boldsymbol{R}^{n}\right)$ for any $\varepsilon>0$, where $q(x)$ satisfies Assumption 2, we have by (4.8) and (4.9)

$$
\begin{aligned}
& \liminf _{s \rightarrow 0} s^{\varepsilon-\frac{1}{2}} \int_{S_{s}}|q(x)||u(x)|^{2} d S \\
& \quad=\liminf _{s \rightarrow 0} s \int_{S_{s}}\left(|q(x)||x|^{\varepsilon-\frac{3}{2}}\right)|u(x)|^{2} d S=0,
\end{aligned}
$$

(4.11) $\left.\liminf _{s \rightarrow 0} s^{2 \varepsilon-2} \int_{S_{s}} i u(x)\right|^{2} d S=\liminf _{s \rightarrow 0} s \int_{S_{s}}|x|^{2 \varepsilon-3}|u(x)|^{2} d S=0$,

and

(4.12) $\quad \liminf _{s \rightarrow 0} s^{\varepsilon-\frac{1}{2}} \int_{S_{s}}|D u(x)|^{2} d S=\liminf _{s \rightarrow 0} s \int_{S_{s}}|x|^{\varepsilon-\frac{3}{2} \mid}|D u(x)|^{2} d S=0$.

In (4.10), (4.11) and (4.12), we put $\varepsilon=\frac{\gamma}{2}$. Then by $\gamma>0$, we have $\gamma>\varepsilon-\frac{1}{2}$. Then (4.10), (4.11), (4.12) and (3.37) show the assertion. Q.E.D. 
Lemma 4.2. There exists some $r_{0}>0$ such that

$$
F\left(r_{0}, \alpha_{0} ; \gamma\right)>0
$$

Proof. In (3.8) we put $s=s_{l}$, and let $l$ tend to $\infty$, then by Lemma 4.1 we have

$$
\begin{aligned}
& F\left(r, \alpha_{0} ; \gamma\right)=\int_{B_{r}}\left[2(1-\gamma)\left\{|D v|^{2}-\langle D v, \tilde{x}\rangle^{2}\right\}\right. \\
& \left.\quad+\left\{2 \gamma \lambda+2(1-\gamma) \tilde{q}(x)+\frac{\gamma(1-\gamma)(2-\gamma)}{2|x|^{2}}\right\}|v|^{2}\right]|x|^{-n+\gamma} d x
\end{aligned}
$$

where $B_{r}=\{x|| x \mid<r\}$. Noting that $u(x)$ is a not identically vanishing function, we have the assertion.

Q.E.D.

\section{Lemma 4.3. We have}

$$
\lim _{r \rightarrow \infty} F\left(r, a_{0} ; \gamma\right)>0
$$

Proof. By Lemma 3.4, $F\left(r . \alpha_{0} ; \gamma\right)$ is a monotone increasing function of $r$ in $(0, \infty)$. Then Lemma 4.2 leads us to the assertion.

Q.E.D.

Now we shall prove Theorem 2.2.

Proof of Theorem 2.2. Noting Lemma 4.3, by a method similar to the first part of the proof of Theorem 2.1, we have the assertion. Q.E.D.

Proof of Corollary to Theorem 2.2. Obvious from Theorem 2.2.

Q.E.D.

\section{References}

[1] Agmon, S., Uniqueness results for solutions of differential equations in Hilbert space with applications to problems in partial differential equations, Lectures in differential equations, Vol. II, A. K. Aziz General Editor, Van Nostrand, Mathematical Studies, No. 19, (1969), 125-144.

[2] Agmon, S., Lower bounds for solutions of Schrödinger-type equations in unbounded domains, Proceedings of the International Conference on Functional Analysis and Related Topics, Tokyo, (1969), 216-224.

[3] Agmon, S., Lower bounds for solutions of Schrödinger equations, $J$. d'Anal. Math., 23 (1970), 1-25.

[4] Ikebe, T. and Kato, T., Uniqueness of the self-adjoint extension of singular elliptic 
operators, Arch. Rational Mech. Ann., 9 (1962), 77-92.

[5] Ikebe, T. and Uchiyama, J., On the asymptotic behavior of eigenfunctions of second-order elliptic operators, J. Math. Kyoto Univ. 11 (1971), 425-448.

[6] Kato, T., Growth properties of solutions of the reduced wave equation with a variable coefficient, Comm. Pure Appl. Math., 12 (1959), 403-425.

[ 7 ] Roze, S. N., On the spectrum of a second-order elliptic operator, Mat. Sb., 80 (112) (1969), 195-209 (Russian).

[8] Weidmann, J., On the continuous spectrum of Schrödinger operators, Comm. Pure Appl. Math., 19 (1966), 107-110.

[9] Weidmann, J., The Virial theorem and its application to the spectral theory of Schrödinger operators, Bull. Amer. Math. Soc., 73 (1967), 452-459. 